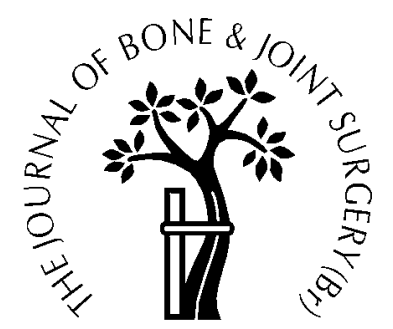

\title{
Strain distribution in the proximal human femur
}

\author{
AN IN VITRO COMPARISON IN THE INTACT FEMUR AND \\ AFTER INSERTION OF REFERENCE AND EXPERIMENTAL \\ FEMORAL STEMS
}

Young-Hoo Kim, J.-S. Kim, S.-H. Cho

From the Joint Replacement Centre of Korea, Seoul, Korea

Gix pairs of human cadaver femora were divided Sequally into two groups one of which received a non-cemented reference implant and the other a very short non-dependent experimental implant. Thirteen strain-gauge rosettes were attached to the external surface of each specimen and, during application of combined axial and torsional loads to the femoral head, the strains in both groups were measured.

After the insertion of a non-cemented femoral component, the normal pattern of a progressive proximal-to-distal increase in strains was similar to that in the intact femur and the strain was maximum near the tip of the prosthesis. On the medial and lateral aspects of the proximal femur, the strains were greatly reduced after implantation of both types of implant. The pattern and magnitude of the strains, however, were closer to those in the intact femur after insertion of the experimental stem than in the reference stem. On the anterior and posterior aspects of the femur, implantation of both types of stem led to increased principal strains E1, E2 and E3. This was most pronounced for the experimental stem.

Our findings suggest that the experimental stem, which has a more anatomical proximal fit without having a distal stem and cortex contact, can provide immediate postoperative stability. Pure proximal loading by the experimental stem in the metaphysis, reduction of excessive bending stiffness of the stem by tapering and the absence of contact between the stem and the distal cortex may reduce stress shielding, bone resorption and thigh pain.

J Bone Joint Surg [Br] 2001;83-B:295-301.

Received 9 April 1999; Accepted after revision 28 October 1999

Y.-H. Kim, MD, Director and President

J.-S. Kim, MD, Orthopaedic Surgeon

S.-H. Cho, MD, Orthopaedic Surgeon

Joint Replacement Centre of Korea, Hae Min General Hospital, 627-3, Ja

Yang 1-Dong, Kwang Jin-Gu, Seoul, Korea 143-191.

Correspondence should be sent to Dr Y.-H. Kim.

(C)2001 British Editorial Society of Bone and Joint Surgery 0301-620X/01/210108 \$2.00
Several studies have shown the changes in strain which occur for different designs of stem, such as those with uncemented fixation ${ }^{1-20}$ and those with a collar. ${ }^{18-20}$ One conclusion from these studies was that a closer proximal fit can produce closer to normal magnitudes and patterns of strain, and a tight distal fit can reduce proximal strains.

The stresses in the bone cannot be measured directly, but measurement of surface strains offers an indirect method of determining the internal forces in bone. There are a number of ways of calculating deformation of bone including finiteelement analysis, 3,15,18,21,22 a photoelastic coating technique, ${ }^{13,20,22-24}$ and strain-gauge analysis. ${ }^{11,19,25-31}$ Although the last technique has practical limitations in the number of gauges that can be used, which precludes visualisation of the continuous strain pattern over the surface of the bone, it is accurate for the measurement of local forces.

Our aim was to use strain-gauge rosettes to measure the principal strains and the pattern of strain in the intact proximal femur, and the changes which occur in these after insertion of a non-cemented very short femoral stem.

\section{Materials and Methods}

Intact femora. We used six pairs of intact, fresh adult human cadaver femora for the biomechanical tests. There were three males and three females with a mean age of 54 years (41 to 61). The femora were wrapped in towels soaked with saline, frozen at $-20^{\circ} \mathrm{C}$ and then thawed at room temperature before use. They were freed of all muscles and radiographs were taken to exclude pathological lesions. They were then randomly assigned either to group 1 (reference stem) or group 2 (experimental stem).

For the measurement of strain, ten $45^{\circ}$ rosettes (120 $\Omega$ 3/120 RY91; Hottinger Baldwin Me Technik GmbH, Darmstadt, Germany) were bonded to the proximal femur at four levels (A to D), namely at the calcar and 2, 4 and $8 \mathrm{~cm}$ distal to the most caudal part of the femoral head. For each pair of femora, the position of the D rosettes was chosen to correspond to the planned position of the tip of the femoral stem to be inserted into one of the paired femora. At level A one rosette was bonded medially at the calcar and at levels B, C and D rosettes were attached at the medial, anterior, lateral and posterior aspects, respectively (Fig. 1). 


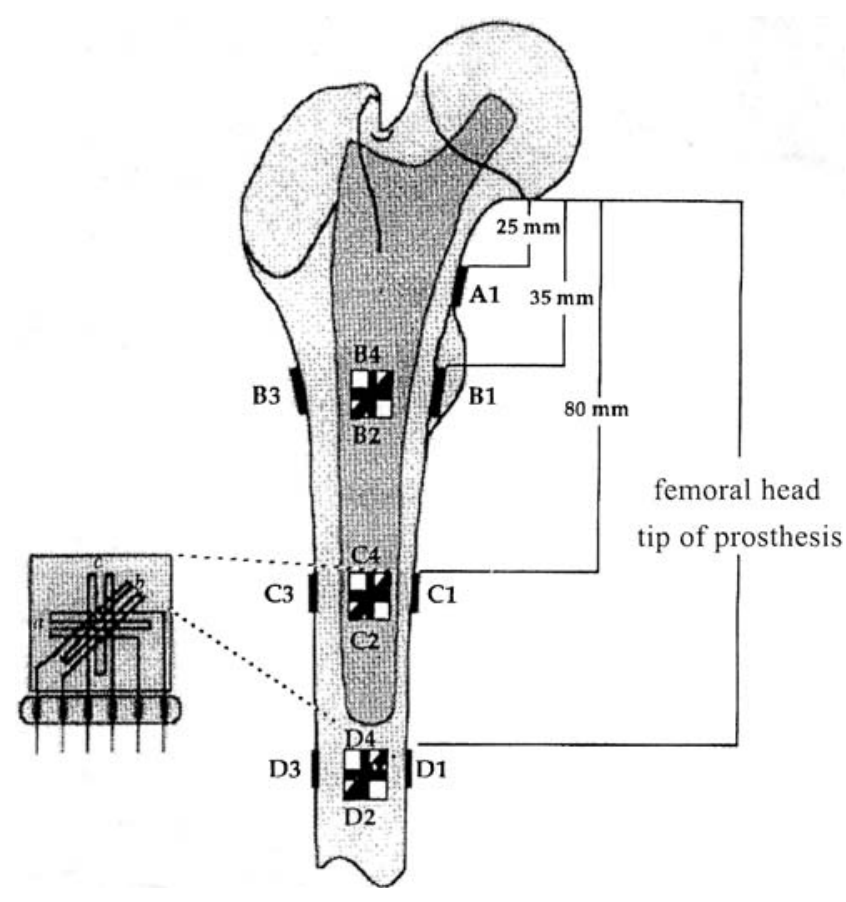

Fig. 1

Diagram of the proximal part of the femur indicating the vertical levels of attachment of the strain-gauge rosettes. The diagram on the left shows a strain-gauge rosette consisting of a polyurethane carrier and three grid elements for the measurement of axial and circumferential strains as well as the strain in the intermediate direction of $45^{\circ}$. The magnitudes and direction of the principal strain can then be computed.

By using the distance from the distal part of the femoral head and the posterior condylar plane as reference, we could reproduce and recover the exact localisation and orientation of the rosettes on the femora.

The selected sites were prepared by first completely removing all soft tissues from the bone. The surface of the bone was smoothed with sand paper (\#100 to 240), degreased with acetone and dried in an $\mathrm{O}_{2}$ stream. An etchant (Multipurpose Etchant; 3M, Minneapolis, Minnesota) was applied to the gauge site for 15 seconds, then rinsed off with saline. After renewed drying the surface was primed (Multipurpose Primer; 3M) and the rosette was bonded using a two-component PMMA adhesive (X-60; Hottinger Baldwin Me Technik $\mathrm{GmbH}$ ). The leads of the gauge were soldered to terminals fixed to the bone immediately adjacent to the rosettes and connected to a signal amplifier (UPM 100; Hottinger Baldwin Me Technik $\mathrm{GmbH})$ by a wire cable.

Finally, the rosette and solder terminal were covered with a waterproof epoxy sealing. Before the actual measurements, the gauges were checked for electrical continuity and for internal resistance $(120 \Omega)$ as recommended by the supplier. Furthermore, we checked that all the electrical terminals had been adequately insulated $(>2 G \Omega)$. One rosette was made up of three strain gauges mounted at $45^{\circ}$ angles on the polyurethane carrier and a gauge was always positioned perpendicular to the longitudinal axis of the femur. In addition to the recording of direct strain from the three gauges, the magnitudes and direction of the principal strain were computed.

After preparation and application of the strain gauges, the femur was placed in a jig which was again mounted in a materials testing system (Lloyd LR10K; Lloyd Instruments Ltd, Hampshire, UK). Distally, the femur was able to rotate around its longitudinal axis and to tilt around its anterior and posterior axis. In this way, non-physiological bending moments on the femur were eliminated. The femur was tilted into $12^{\circ}$ of valgus which corresponds to the physiological inclination during single-leg stance. Load was applied to the femoral head by a lever arm connected to the cross-head of the testing machine. The centre of the cup containing the femoral head was positioned $110 \mathrm{~mm}$ lateral to the load axis and the trochanter strap formed an angle of $75^{\circ}$ to the lever arm, which simulates the pelvis. The trochanter strap was adjustable both in length and position on the lever arm, which was horizontal when maximum axial load was applied to the femoral head. For the simulation of both hip abductors and the iliotibial band a wire extension from the trochanter strap ran over two pulleys mounted on the outer end of the lever arm. The iliotibial band ran distally to be attached $50 \mathrm{~mm}$ lateral to the central axis of the femur and we checked that the strap did not touch the greater trochanter when the femur was bent laterally during loading.

In addition to the application of axial load through the Lloyd cross-head, torsional load was applied to the femur using a weight-and-pulley system acting on transverse crossbars mounted to the cylinder containing the specimen. Thus, the torsional load was applied to the distal femur and the moments were transmitted to the trochanter strap and the acetabular cup, which prevented the femur from rotating. By means of a load cell on the acetabular cup the torsional forces acting on the head could be monitored. In addition to the acetabular cup, the trochanter/iliotibial band and the torsional loading system also had load cells allowing continuous monitoring of the forces.

The femur was preloaded with an axial force of $900 \mathrm{~N}$, then unloaded and the strain gauges were adjusted to zero. Loading was applied to the intact femur to simulate a singleleg stance as well as stair-climbing. One measurement from each of the strain gauges was stored during the plateau phase of each of the stages. The resultant force on the femoral head was not measured directly. Based on the static loads on the proximal femur, however, computations show that the resultant load on the head was approximately $225 \%$ of the axial load. Thus, the resultant load on the intact femoral head during maximum axial and torsional loading was at least $2025 \mathrm{~N}$. The intact femur was tested by applying an axial load of up to $900 \mathrm{~N}$ whereas the operated femur was tested using a maximum axial force of $1200 \mathrm{~N}$. We did not use the $1200 \mathrm{~N}$ load on the intact femur because this greatly exceeds the physiological loads in the hip during normal activities and could result in breakage of the bone. 


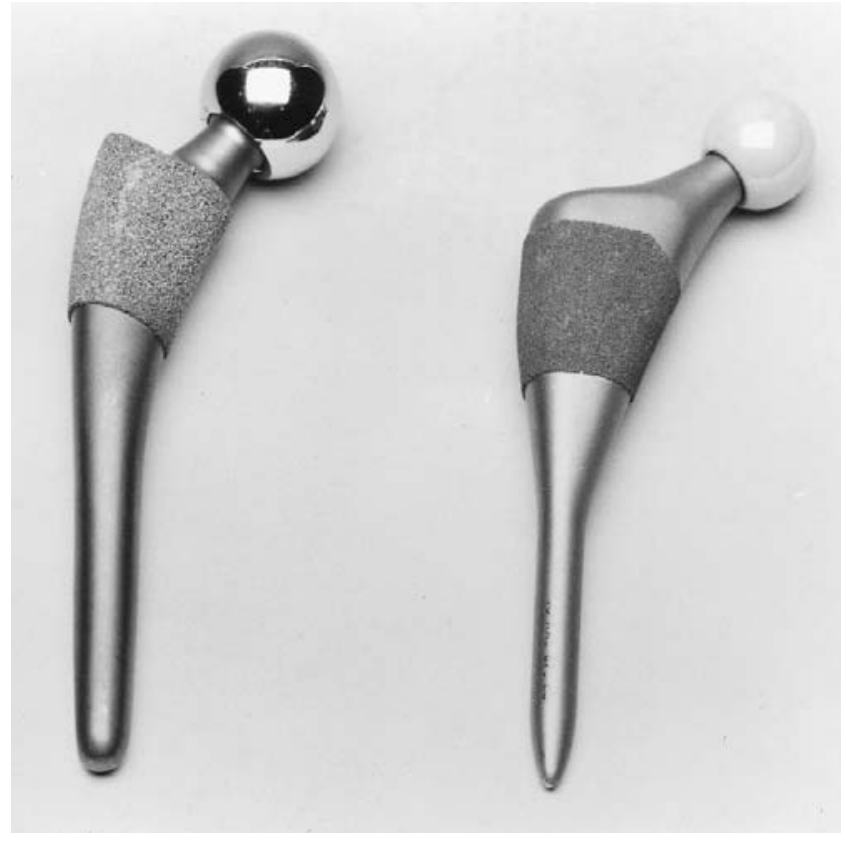

Fig. 2a

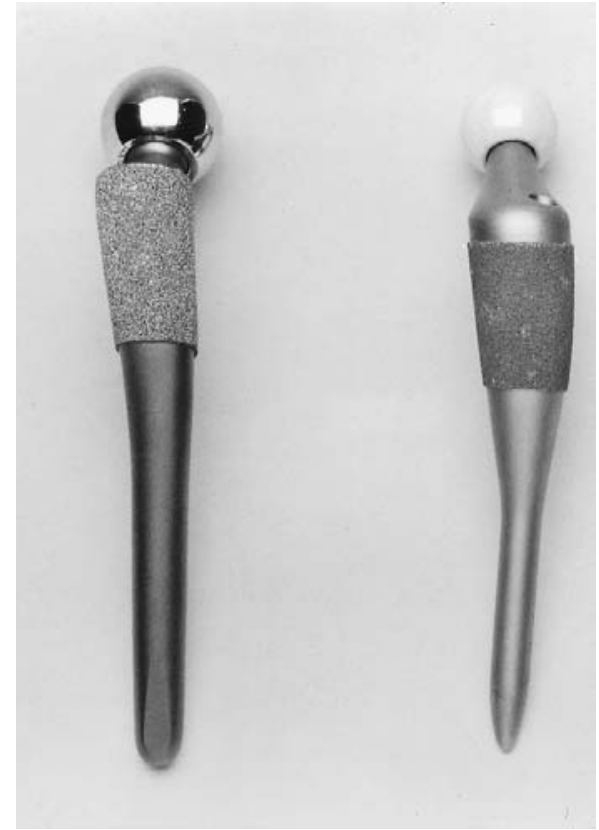

Fig. $2 b$

Figure $2 \mathrm{a}-$ Anteroposterior view of a reference (left) and an experimental stem (right). The diameter of the experimental stem is markedly reduced distally and the medial part of the proximal stem is more curved, which probably ensures a closer cortical fit in the calcar region. The lateral part of the stem is designed to fit the lateral flare of the femur. Figure $2 b-$ Lateral view of $a$ reference (left) and an experimental stem (right). The diameter of the experimental stem is markedly reduced and the anteroposterior diameter of the proximal stem is substantially increased compared with the reference stem.

Insertion of the femoral components. A reference noncemented anatomic stem (DePuy, Leeds, UK) was used for the proximal and distal implant and an experimental noncemented anatomic stem (DePuy) for the proximal fit only. Both were made of TiVaAl alloy. They had an anatomic design and six sizes were available for the left and right femur. The proximal one-third of the stem had a porous coating of pure titanium beads (Fig. 2). The stems had a 12/14 taper and were used with cobalt-chromium modular heads available in five different neck lengths $(+1.5$ to 15.5).

The design of the experimental stem had some differences from that of the reference stem; the diameter was markedly reduced distally, and the medial part of the proximal stem was more curved, which probably ensured a closer cortical fit in the calcar region. Moreover, the lateral part of the stem was designed to fit the lateral flare of the femur. Finally, the anteroposterior diameter of the proximal stem was substantially increased compared with the reference stem. Thus, the design characteristics of the experimental stem assisted proximal stability and load transmission (Fig. 2).

After the distribution of strain had been determined in the intact femur, the femoral head was carefully removed by sawing through the neck, and the medullary canal reamed as at surgery. The position of the femoral prosthesis in the medullary canal was checked by radiological examination. The tests described for the intact femur were repeated under the identical conditions of position and load.
Loads were applied to the head of the femoral component through an appropriate polyethylene acetabular cup. Experimental and reference stems were compared in terms of their effects on the distribution of strain in the proximal end of the femur.

The principal strain was calculated as follows:

$E 1(E 2)=\frac{E a+E c}{2}-\frac{1}{2}(E a 2+E c 2)+E b(E b+E a-E c)$

where E1 is a large principal strain (usually tension), E2 is a large principal strain (usually compression), Ea is the strain in gauge element $\mathrm{a}, \mathrm{Eb}$ is the strain in gauge element $\mathrm{b}, \mathrm{Ec}$ is the strain in gauge element c, Ed is the strain in gauge element d, and the principal shear strain (E3) is E1E2.

\section{Results}

Intact femora. Figure 3 shows representative strain data for the proximal part of the 12 intact femora when subjected to a force of $900 \mathrm{~N}$ applied vertically and $15 \mathrm{Nm}$ torsional load with the femoral shaft in $12^{\circ}$ of adduction. Strains were increased from proximal to distal in the intact femora under load, and the highest values were in area D. They were higher in compression on the concave side than in tension on the convex side at all levels and were greater in the coronal (Fig. 3c) than in the sagittal plane (Fig. 3b). Since the tensile strains on the lateral side were consistent 


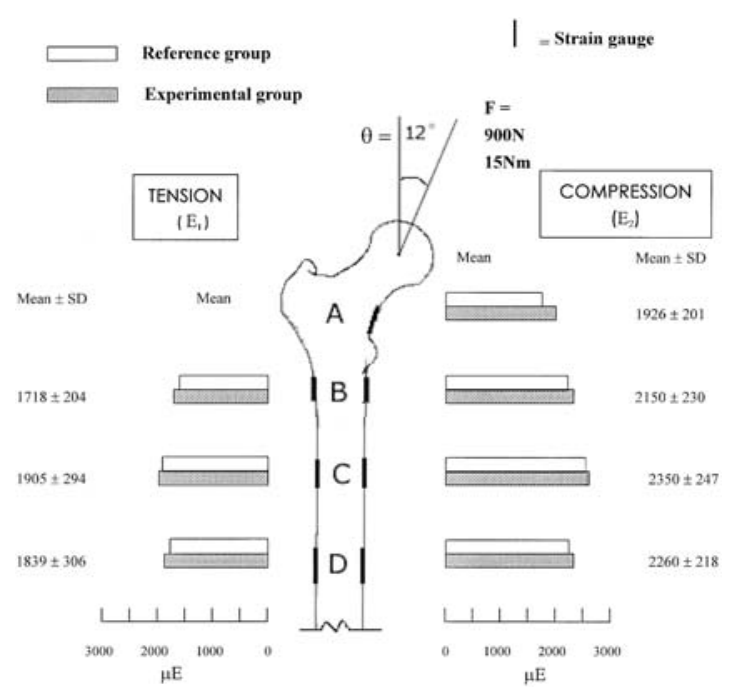

Fig. 3a

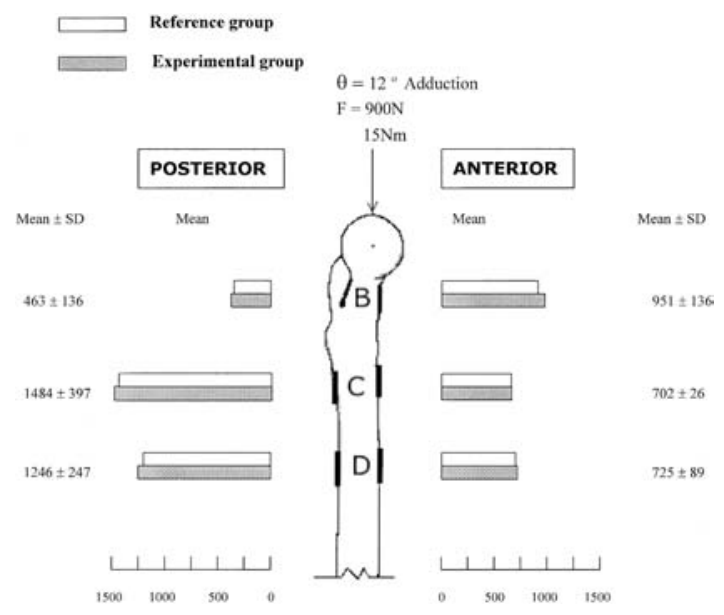

Fig. 3c

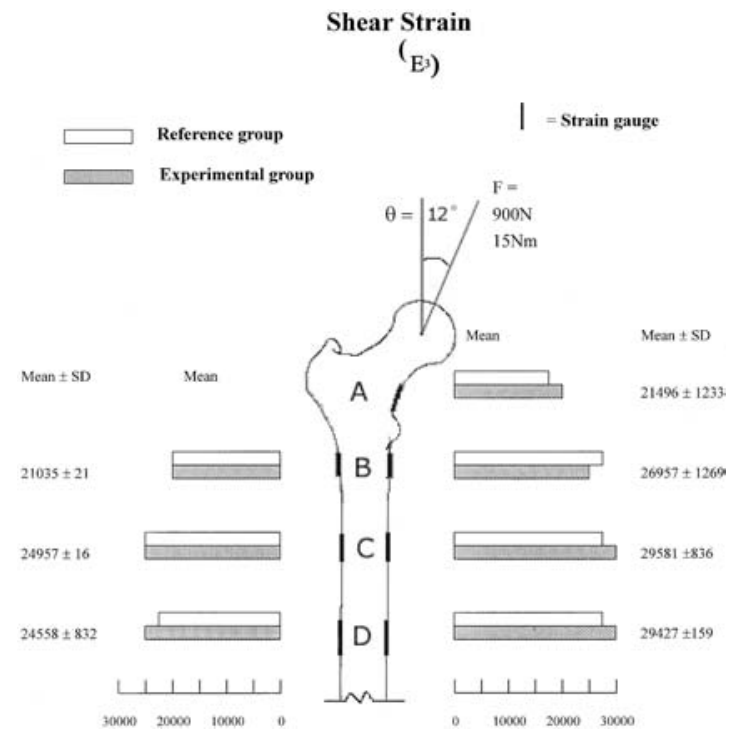

Fig. 3e

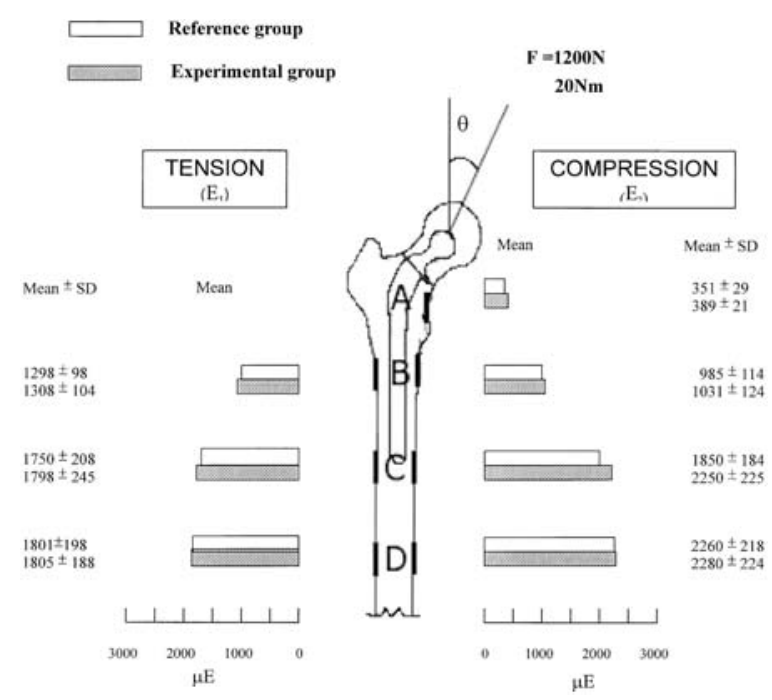

Fig. 3b
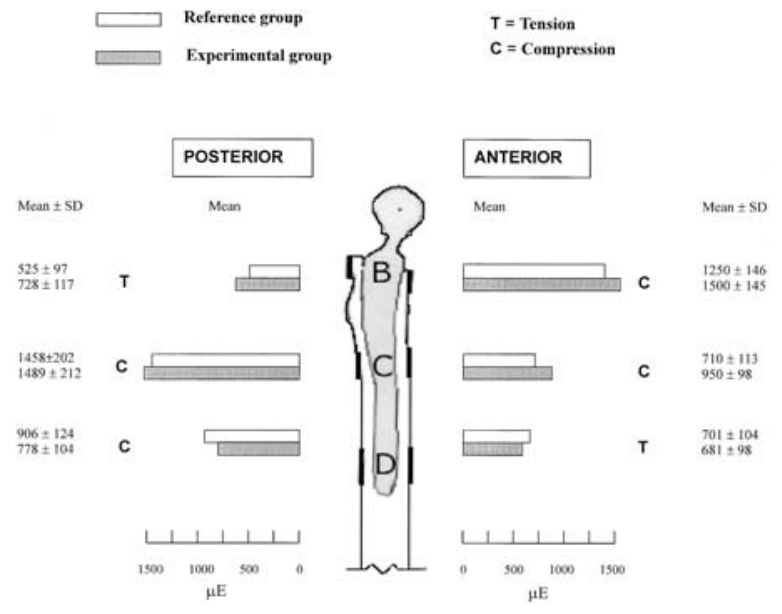

Fig. 3d

\section{Shear Strain}

(E)

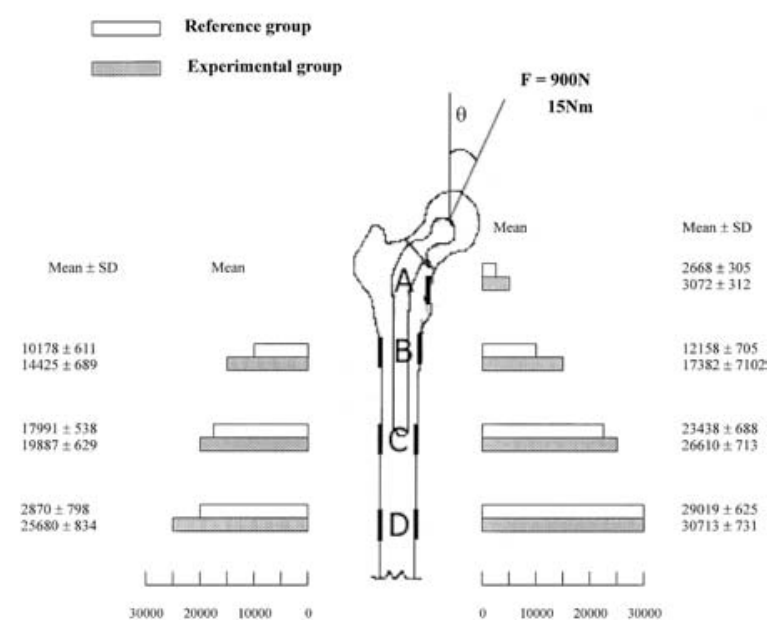

Fig. 3f 
Shear Strain

( $\left.\mathrm{E}^{3}\right)$

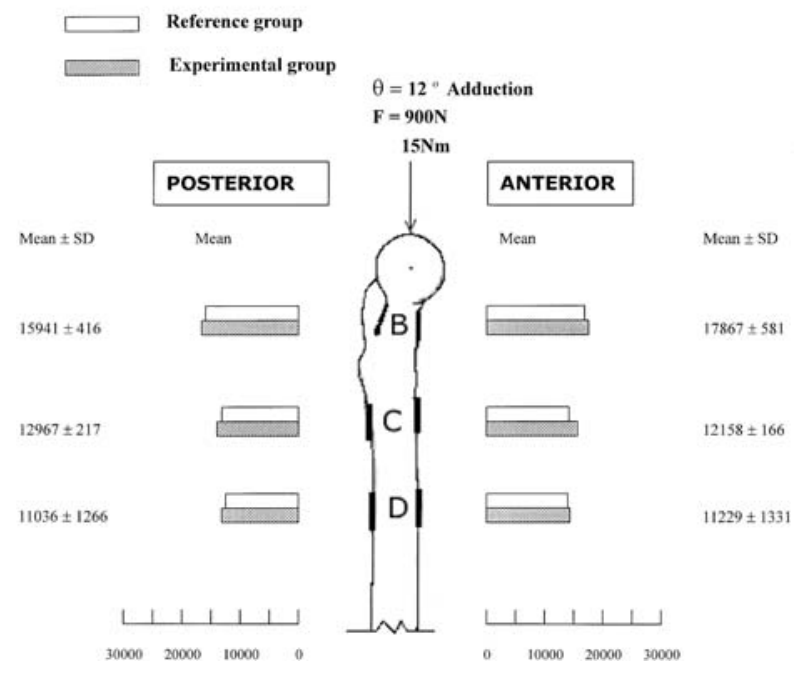

Fig. $3 g$
Shear Strain

$\left(\mathrm{E}_{3}\right)$

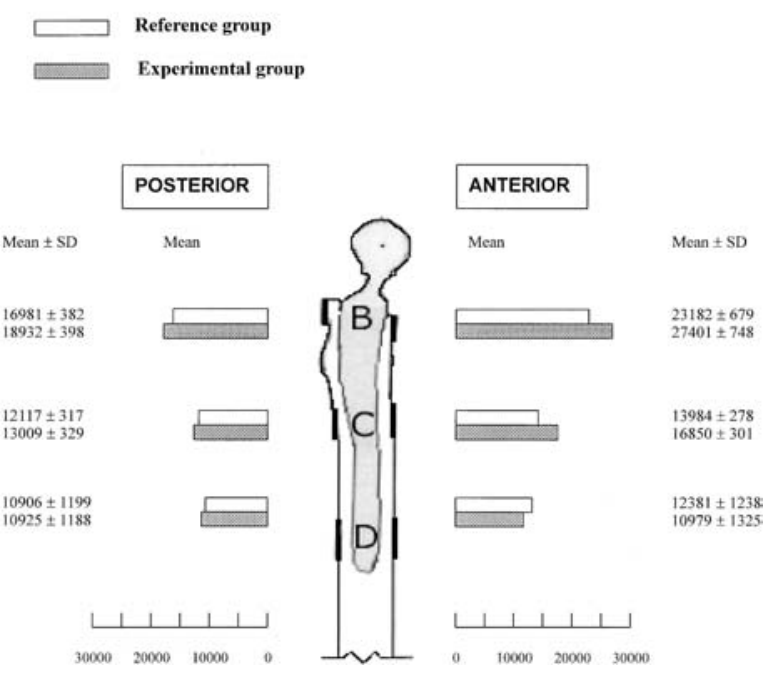

Fig. $3 \mathrm{~h}$

Bar graphs (coronal plane) showing representative data on the distribution of strains in the 12 intact and 12 operated human femora. For the former, the angle of load was $12^{\circ}$ and the magnitude of load was $900 \mathrm{~N} / 15 \mathrm{Nm}$ and for the latter $12^{\circ}$ and $1200 \mathrm{~N} / 20 \mathrm{Nm}$, respectively. Figure $3 \mathrm{a}$ - The distribution of strain in the lateral and medial cortices (frontal plane). The pattern of increasing compressive and tensile strain progressing from proximal to distal is present in all femora. Figure $3 b$ - In general the strain (coronal plane) in the calcar area after insertion of the femoral components is sharply reduced, but the loss is greater in the reference stems. At the level of the calcar femorale, when the femoral components were in situ the compressive strains were significantly different from those in the intact femur at the $95 \%$ level of confidence. At the other levels, the strains with the implants in place are not significantly different from those in the intact femur. With the components in place, the actual levels of strain at the tip of the stem were nearly normal. Figure $3 \mathrm{c}$ - The distribution of strain in the anterior and posterior cortices (sagittal plane). The strains in the anterior and posterior sites are substantially less in general than those at the medial and lateral sites. The strain is also greater in the posterior surface of the proximal part of the femoral shaft than in either the anterior or the posterior surface of the femoral neck. Figure $3 \mathrm{~d}$ - The distribution of strain (sagittal plane) shows that there is a significant increase in strains on the anterior and posterior aspects of the femur at level B after insertion of the stem. At the other level, the strains with the implants in place are not significantly different from those in the intact femur. Figure $3 \mathrm{e}-$ The distribution of shear strain in the lateral and medial cortices (frontal plane) which shows increasing shear strain progressing from proximal to distal in all femora. Figure $3 \mathrm{f}$ - In general the shear strain (coronal plane) in the calcar area after insertion of the femoral components is sharply reduced, but the loss is greater with the reference stems. At the level of the calcar femorale, when the femoral components were in situ the shear strains are significantly different from those in the intact femur at the $95 \%$ level of confidence. At the other levels, the strains with the implants in place are less significantly different from the shear strain in the intact femur. With the components in place, the actual levels of shear strains at the tip of the stem are nearly normal. Figure $3 \mathrm{~g}$ - The distribution of shear strain in the anterior and posterior cortices (sagittal plane). The shear strains in the anterior and posterior sites are less in general than those at the medial and lateral sites. The shear strain is greater in the anterior and posterior surface of the femoral neck than in either the anterior or the posterior surface of the femoral shaft. Figure $3 \mathrm{~h}$ - The distribution of shear strain (sagittal plane) shows that there is a significant increase in strains on the anterior and posterior aspects of the femur at level B after stem insertion. At the other levels, the strains with the implants in place are not significantly different from the strains in the intact femur.

with previous mathematical analysis, ${ }^{29}$ the compressive strains on the medial side were always greater in the subtrochanteric area than in the area of the calcar femorale. Shear strains were higher on the concave side than on the convex side at all levels and were greater in the coronal (Fig. 3e) than in the sagittal plane (Fig. 3g). The shear strains over the medial side were always greater in the subtrochanteric area than in the area of the calcar femorale.

The distal portion of the posterior part of the neck of the femur was under tension in these loading conditions and the posterior part of the femur distal to the lesser trochanter showed compressive strain, as expected from the normal concavity of the posterior aspect of the femur. In the anterior surface of the femur, compressive strain was present in the neck and subtrochanteric area but there were tensile strains in the area of the isthmus of the femur and distal to it. The strain in the anterior aspect of the neck was appreciably larger than in the anterior surfaces of the rest of the proximal part of the femur (Fig. 3c). The shear strain in the anterior aspect of the neck was also appreciably larger than that in the anterior surfaces of the rest of the proximal part of the femur (Fig. 3g).

Insertion of the femoral components. For both groups the mean value of E1, E2 and E3 was computed. The data are presented as horizontal bar diagrams indicating the mean values of E1, E2 and E3 at each of the 13 locations of strain measurement on the proximal femur. For each of the loading configurations one graph shows the strains on the medial and lateral aspects and the other on the anterior and posterior aspects of the femur.

We used Student's $t$-test for paired samples to analyse the difference in the mean principal strain in both groups of operated femora. A p value of less than 0.05 indicated statistically significant differences.

The strain pattern or distribution of strain was markedly 
changed after insertion of a femoral implant. As would be expected in the proximal and medial aspects of the femur (level A) there was a decrease in the principal compressive strain (E2) $(82 \%$ for the reference and $80 \%$ for the experimental group, $\mathrm{p}=0.3017)$ and in shear strain $(\mathrm{E} 3)(88 \%$ and $86 \%$, respectively, $\mathrm{p}=0.3107)$. These differences were not significant (Figs 3b and 3f).

After insertion of the femoral component, the pattern of strain going from the area of the calcar femorale distally to the tip of the stem, was similar to that in the intact femur. At level $\mathrm{B}$, which is just below the lesser trochanter, there was a decrease in principal compressive strain E2 (64\% for the reference and $39 \%$ for the experimental group, $\mathrm{p}=0.0065)$ and in shear strain E3 (55\% and 36\%, respectively, $\mathrm{p}=0.0171)$. At levels $\mathrm{C}$ and $\mathrm{D}$ the change in $\mathrm{E} 1, \mathrm{E} 2$ and E3 was less pronounced in both groups of femora. At level C, there was a decrease in strain E2 $(22 \%$ and $4 \%$, respectively, $\mathrm{p}=0.0424)$ and in strain $\mathrm{E} 3(21 \%$ and $10 \%$, respectively, $\mathrm{p}=0.0448)$. At level $\mathrm{D}$ the principal E1, E2 and E3 strains were almost the same as in the intact femora for both groups of implanted specimens (Figs $3 \mathrm{~b}$ and $3 \mathrm{f}$ ). An unexpected observation was an increase in the strains on the anterior and posterior aspects of the femur at level B after insertion of the stem. There was an increase in tension strain E1 on the posterior aspect of the femur (35\% for the reference and $57 \%$ for the experimental group, $\mathrm{p}=0.0012$ ) and shear strain E3 (6\% and 16\%, respectively, $\mathrm{p}=0.0431$ ). On the anterior aspect of the femur at level $\mathrm{B}$, there was an increase in compressive strain E2 $(31 \%$ and $58 \%$, respectively, $\mathrm{p}=0.0009)$ and in shear strain E3 (30\% and 53\%, respectively, $\mathrm{p}=0.0032$ ). At level $\mathrm{C}$, the compression strain $\mathrm{E} 2$ on the anterior aspect was almost the same as that in the intact femur for the reference group. For the experimental group the increase was $35 \%(\mathrm{p}=0.0453)$. The shear strain E3 on the anterior aspect was increased slightly $(15 \%)$ in the reference group and the increase in the experimental group was $39 \%(\mathrm{p}=0.0452)$. The compression strain E2 and the shear strain E3 in the posterior aspect of level C were almost the same as those in the intact femur for both groups $(p=0.3681)$. These findings indicate that the insertion of the stem led to increased deformation in level B, and presumably higher stresses in the experimental group, on the anterior and posterior aspects of the femur (Figs 3d and $3 h)$.

On the lateral aspect of the femur, as on the medial side, a decrease in tensile strains was observed. At level B, there was a decrease in principal strain E1 (25\% for the reference and $24 \%$ for the experimental group, $\mathrm{p}=0.3789$ ) and in shear strain E3 (52\% and 31\%, respectively, $\mathrm{p}=0.3687)$. At level $\mathrm{C}$, the decrease in the principal tensile strain $\mathrm{E} 1$ was $8 \%$ and $6 \%$, respectively $(\mathrm{p}=0.7658)$. The decrease in shear strain E3 was $28 \%$ for the reference group and $20 \%$ for the experimental group $(p=0.3891)$. At level $D$ the changes in tensile strain E1 and shear strain E3 were minor for both groups $(p=0.9322)$.

Using the intact femora as a reference, insertion of the prosthesis in the reference group led to an overall reduction in strain in levels A and B of $37 \%$ whereas for femora in the experimental group the reduction was $22 \%(\mathrm{p}=0.005)$. This difference seems to be due to a higher level of compressive and shear strains in the proximal region in the experimental femora. In addition, it is possible that the experimental stem was more able to transmit forces to the proximal femur than the reference stem. This conclusion seems at least to be valid for the anterior and posterior aspects of the femur at level B when the strains were higher in the experimental femora.

\section{Discussion}

Oh and Harris ${ }^{28}$ reported that strains decreased from proximal to distal in the intact femora under load, and that the highest values were in the calcar area. By contrast, we have found that strain increased from proximal to distal in the intact femora under load and the highest values were in areas $\mathrm{C}$ and $\mathrm{D}$. This finding is consistent with previous mathematical analysis. 22,30

One of the important findings in our study was the reduction in compressive, tensile and shear strains in the proximal part of the femur which results from insertion of a femoral component, regardless of type. The strains in the calcar area were greatly reduced in both groups. Rapid disuse atrophy of bone as a sequel to reduced stress has been well documented, both in the skeleton as a whole ${ }^{31}$ and as a local phenomenon. ${ }^{29}$ The measured severe decrease in longitudinal and shear stresses in the region of the calcar femorale found in our study suggest the strong probability, as reported by Charnley and Cupic, ${ }^{32}$ that this is at least in part the result of disuse atrophy.

$\mathrm{Oh}$ and Harris ${ }^{28}$ believed that the large reduction in strain in the area of the calcar femorale must be associated with a substantial increase in bone resorption. In our study, although the compressive, tensile, and shear strains in the proximal femur were reduced in the coronal plane after the insertion of both anatomical femoral stems, they were increased in the sagittal plane. An experimental stem with a more anatomical fit in the proximal femur had more increased strain $(58 \%)$ than the reference stem $(31 \%)$. The increased strains in the proximal femur in the experimental stem may be attributed to the increased proximal hoop stress. Increased strains in the anterior and posterior aspects of the proximal femur may lead to stress concentration over a short metaphyseal segment which could increase interface stresses and compromise the biological bone ingrowth in this region. We believe, however, that these increased strains would reduce the disuse atrophy and would not compromise the bone ingrowth in the region.

Maloney et $\mathrm{al}^{33}$ found that the maximum bone loss for the cemented and cementless specimens did not occur in the proximal metaphysis. The maximum cortical bone loss was at the middle level for the cemented femora and at the mid-proximal and middle levels for the cementless. 
Although the strains around the mid-region of the stem in our study are still more than half of the normal, those in the experimental stem are higher than in the reference stem. These higher strains around the experimental stem can reduce the bone resorption in the mid-region of the femur.

The strains in the femur at levels $\mathrm{C}$ and $\mathrm{D}$ after insertion of the femoral component are within the range of those experienced normally by that segment of the femur. Thus, these normal levels of strain may contribute to normal bone remodelling around the stem.

These static studies in vitro showing the potential benefits from an experimental proximal stem suggest that further work should be done on ways to obtain broad contact between the proximal medial surface of the femoral component and the proximal medial surface of the femur. The weakness of our study is that the model reflects the situation soon after implantation. There is no biological fixation of the prosthesis to surrounding bone. This may alter the pattern of strain distribution.

Our study may have a clinical application. An experimental stem which has a more anatomical fit without having contact with a distal stem and cortex can provide immediate postoperative stability. Pure proximal loading by the experimental stem in the metaphysis, reduction of excessive bending stiffness of the stem by tapering the stem and absent contact between the stem and the distal cortex may reduce stress shielding bone resorption and thigh pain.

One or more of the authors have received or will receive benefits for personal or professional use from a commercial party related directly or indirectly to the subject of this article.

\section{References}

1. Brown IW, Ring PA. Osteolytic changes in the upper femoral shaf following porous-coated hip replacement. J Bone Joint Surg $[\mathrm{Br}]$ 1985;67-B:218-21.

2. Hille E, Shulitz KP, Gipperich J, Dettman B. Experimental stressinduced changes in growing long bones. Int Orthop 1988;12:309-15.

3. Huiskes R, Weinans H, Dalstra M. Adaptive bone remodelling and biomechanical design considerations for non-cemented total hip arthroplasty. Orthopedics 1989;12:1255-67.

4. Maloney WJ, Jasty M, Burke DW, et al. Biomechanical and histologic investigation of cemented total hip arthroplasties: a study of autopsy-retrieved femurs after in vivo cycling. Clin Orthop 1989;249:129-40

5. Capello WN. Fit the patient to the prosthesis: an argument against the routine use of custom hip implants. Clin Orthop 1989;249:56-9.

6. Hua J, Walker PS. Relative motion of hip stems under load: an in vitro of symmetrical, asymmetrical and custom asymmetrical designs. J Bone Joint Surg [Am] 1994;76-A:95-103.

7. Knessl J, Gschwend N, Scheier H, Munzinger U. Comparative study of cemented and cementless hip prostheses in the same patient. Arch Orthop Trauma Surg 1989;108:276-8.

8. Morscher EW. Cementless total hip arthroplasty. Clin Orthop 1983;181:76-91.
9. Noble PC, Alexander JW, Lindahl LJ, et al. The anatomic basis of femoral component design. Clin Orthop 1988;235:148-65.

10. Schimmel JW, Huiskes R. Primary fit of the Lord cementless tota hip: a geometric study in cadavers. Acta Orthop Scand 1988;59:638-42.

11. Walker PS, Schneeweis, Murphy S, Nelson P. Strain and micromotions of press-fit femoral stem prostheses. $J$ Biomech 1987;20:693-702.

12. Walker PS, Robertson DD. Design and fabrication of cementless hip stems. Clin Orthop 1988;235:25-34.

13. Zhou XM, Walker PS, Robertson DD. Effect of press-fit femoral stems on strains in the femur: a photoelastic coating study. J Arthroplasty 1990;5:71-82.

14. Diegal PD, Daniels AU, Dunn HK. Initial effect of collarless stem stiffness on femoral bone strain. J Arthroplasty 1989;4:173-8.

15. Huiskes R, Weinans $\mathbf{H}$, van Rietbergen $\mathbf{B}$. The relationship between stress shielding and bone resorption around total hip stems and the effects of flexible materials. Clin Orthop 1992;274:124-34.

16. Fyhrie DP, Carter DR, Schurman DJ. Effects of ingrowth, geometry, and material on stress transfer under porous-coated hip surface replacements. J Orthop Res 1988;6:425-33.

17. Heimke G, Griss P, Werner E, Jentschura G. The effects of mechanical factors on biocompatibility tests. J Biomed Eng 1981;3:209-13

18. Crowninshield RD, Brand RA, Johnston RC, Pedersen DR. An analysis of collar function and the use of titanium in femoral prostheses. Clin Orthop 1981;158:270-7.

19. Holmberg PD, Bechtold JE, Sun BN, Kyle RF, Gustilo RB. Strain analysis of a femur with long stem press fit prosthesis. Trans Orthop Res Soc 1986;11:336.

20. Russel K, Whiteside L. Photoelastic stress analysis of the proximal femur after collared and collarless total hip replacement. Orthop Trans 1986;10:399.

21. Rohlmann A, Mossner U, Bergmann G, Kolbel R. Finite elementanalysis and experimental investigation in a femur with hip endoprosthesis. J Biomech 1983;16:727-42.

22. McNeice GM, Eng P, Amstutz HC. Finite element studies in hip reconstruction. Procs the 5th International Congress of Biomechanics. University of Jyraskyla, 1975:394-405.

23. Evans FG, Lissner HR. Stresscoat deformation studies of femur under static vertical loading. Anat Rec 1948;100:159-90.

24. Kalén R. Strains and stresses in the upper femur studied by the Stresscoat method. Acta Orthop Scand 1961;31:103-13.

25. Murphy SB, Walker PS, Schiller AL. Adaptive changes in the femur after implantation of an Austin Moore prosthesis. J Bone Joint Surg [Am] 1984;66-A:437-43.

26. Andriacchi TP, Galante JO, Belytschko TB, Hampton S. Stress analysis of the femoral stem in total hip prostheses. J Bone Joint Surg [Am] 1976;58-A:618-24.

27. Koch JC. The laws of bone architecture. Am J Anat 1917; 21: 177-298.

28. Oh I, Harris WH. Proximal strain distribution in the loaded femur: an in vitro comparison of the distributions in the intact femur and after insertion of different hip-replacement femoral components. $J$ Bone Joint Surg [Am] 1978;60-A:75-85.

29. Rybicki EF, Simonen FA, Weis EB Jr. On the mathematical analysis of stress on human femur. J Biomech 1972;5:205-15.

30. Heaney RP. Radiocalcium metabolism in disuse osteoporosis in man. Am J Med 1962;33:188-200.

31. Burkhart JM, Jowsey J. Parathyroid and thyroid hormones in the development of immobilization osteoporosis. Endocrinology 1967;81:1053-62.

32. Charnley J, Cupic Z. The nine and ten year results of the low-friction arthroplasty of the hip. Clin Orthop 1973;95:9-25.

33. Maloney WJ, Sychterz C, Bragdon $\mathbf{C}$, et al. Skeletal response to well fixed femoral components inserted with and without cement. Clin Orthop 1996;333:15-26. 